\title{
PPAPDC1B and WHSC1L1 Are Common Drivers of the 8p11-12 Amplicon, Not Only in Breast Tumors But Also in Pancreatic Adenocarcinomas and Lung Tumors
}

Sardar F. Mahmood,$^{* \dagger}$ Nadège Gruel, ${ }^{\dagger \ddagger \S}$ Rémy Nicolle, ${ }^{* \dagger}$ Elodie Chapeaublanc, ${ }^{* \dagger}$ Olivier Delattre, ${ }^{\dagger \S}$ François Radvanyi, ${ }^{{ }^{\dagger \dagger}}$ and Isabelle Bernard-Pierrot ${ }^{\star \dagger}$

From the National Center for Scientific Research (CNRS), UMR 144,* the Research Center, ${ }^{\dagger}$ the Translational Research Department, ${ }^{\ddagger}$ and the National Institute of Health and Medical Research (INSERM), U830, ${ }^{\S}$ Institut Curie, Paris, France

Accepted for publication

July 9, 2013.

Address correspondence to Isabelle Bernard-Pierrot, Ph.D., UMR 144 Centre National de la Recherche Scientifique/Institut Curie, 26 rue d'Ulm, 75248 Paris Cedex 05, France. E-mail: ibernard@curie.fr.

\begin{abstract}
Amplification of the 8p11-12 chromosomal region is a common genetic event in many epithelial cancers. In breast cancer, several genes within this region have been shown to display oncogenic activity. Among these genes, the enzyme-encoding genes, PPAPDC1B and WHSC1L1, have been identified as potential therapeutic targets. We investigated whether PPAPDC1B and WHSC1L1 acted as general driver genes, thereby serving as therapeutic targets in other tumors with 8p11-12 amplification. By using publicly available genomic data from a panel of 883 cell lines derived from different cancers, we identified the cell lines presenting amplification of both WHSC1L1 and PPAPDC1B. In particular, we focused on cell lines derived from lung cancer and pancreatic adenocarcinoma and found a correlation between the amplification of PPAPDC1B and WHSC1L1 with their overexpression. Loss-of-function studies based on the use of SiRNA and shRNA demonstrated that PPAPDC1B and WHSC1L1 played a major role in regulating the survival of pancreatic adenocarcinoma and small-cell lung cancer-derived cell lines, both in anchorage-dependent and anchorage-independent conditions, displaying amplification and overexpression of these genes. We also demonstrated that PPAPDC1B and WHSC1L1 regulated xenograft growth in these cell lines. Finally, quantitative RT-PCR experiments after PPAPDC1B and WHSC1L1 knockdown revealed exclusive PPAPDC1B and WHSC1L1 gene targets in small-cell lung cancer and pancreatic adenocarcinoma-derived cell lines compared with breast cancer. (Am J Pathol 2013, 183: 1634-1644; http://dx.doi.org/10.1016/j.ajpath.2013.07.028)
\end{abstract}

Oncogene activation, through gene amplification resulting in overexpression, contributes to malignant transformation in many solid tumors. ${ }^{1,2}$ Functional genomics can be used to identify driver genes within the amplified region, which also encompasses passenger genes, making it possible to identify drug targets. The 17q12 amplicon has been extensively characterized in breast cancer and highlights the potential of tumor genomics. Indeed, the ERBB2 receptor gene on chromosome $17 \mathrm{q} 12$ can be effectively targeted in breast cancers, in which it is amplified by specific treatments, such as trastuzumab (Herceptin, Roche SAS, Boulogne-Billancourt, France). ${ }^{3-5}$

Chromosomal arm 8p rearrangement is a common genetic event in many epithelial cancers, as reviewed by Birnbaum et al. ${ }^{6}$ Amplification of the 8p11-12 chromosomal region is common in many cancers, including breast cancer, in which it has been reported to occur in approximately $15 \%$ of cases and correlated with histological grade, Ki-67 proliferation index, and poor prognosis. $^{7-12}$ Amplification of the 8p11-12 region has also been reported in lung, ${ }^{13}$ bladder, ${ }^{14}$ and pancreatic ${ }^{15,16}$ cancers.

Supported by the Ligue Nationale Contre le Cancer (to Oncologie Moléculaire, accredited team), the Institut Curie, the Centre National de la Recherche Scientifique, the Institut National de la Santé et de la Recherche Médicale, INCa (IVOiRE) grants, the Higher Education Commission of Pakistan grant for PhD research (S.F.M.), and an Association pour la Recherche sur le Cancer France grant (S.F.M.).

The results published herein are, in part, based on data generated by The Cancer Genome Atlas pilot project established by the National Cancer Institute and the National Human Genome Research Institute.

N.G. and R.N. contributed equally to this work. 
Based on the significant correlation between gene copy number and gene expression, followed by functional studies, several oncogenes have been identified within the 8p11-12 amplicon in breast cancer: ZNF703, LSM1, BAG4, C8orf4, FGFR1, WHSC1L1, and PPAPDC1B. ${ }^{12,17-22}$ Among these, WHSC1L1 has been shown to have oncogenic properties in lung cancer cell lines displaying 8p11-12 amplification, ${ }^{13}$ and FGFRl has been shown to be overexpressed when amplified and to play an oncogenic role in bladder and colorectal cancers. ${ }^{14,23-25}$ In addition to FGFRl, two genes from the 8p11-12 amplicon, WHSC1L1 and PPAPDC1B, are particularly interesting in terms of their potential as treatment targets. Indeed, these two genes encode enzymes, which could be targeted by small molecules blocking the catalytic site. Wolf-Hirschorn syndrome candidate 1-like 1 (WHSClL1; alias NSD3) is a member of the nuclear receptor-binding SET domain (NSD) family of histone methyl transferases. ${ }^{14,23-27}$ The use of epigenetic targets, including histone methyltransferase, histone deacetylase, and DNA methyltransferase, is emerging as a potential method of cancer treatment, as reviewed by Morishita and di Luccio. ${ }^{28}$ Phosphatidic acid phosphatase type 2-containing 1B (PPAPDC1B) is a lipid phosphatase. ${ }^{29}$ There is also growing interest in the use of phosphatases as therapeutic targets in various diseases, including diabetes, obesity, and cancer. $^{30-32}$ In addition, PPAPDC1B is a transmembrane protein and could, therefore, be targeted by antibodies. WHSC1L1 was first implicated in cancers with 8p11-12 amplification in the lung. ${ }^{13}$ We subsequently showed that both PPAPDCIB and WHSCILI were involved in the survival and transformation of breast cancer cell lines with 8p11-12 amplification. ${ }^{17}$ This involvement of WHSC1L1 in breast cancer was recently confirmed. ${ }^{22}$

In this study, we investigated whether these two genes, WHSC1L1 and PPAPDC1B, acted as general driver genes and could be considered as suitable treatment targets in tumors with 8p11-12 amplification other than breast cancers. We identified cell lines presenting amplifications of both WHSC1L1 and PPAPDC1B within the 8p11-12 region derived from cancers at various primary sites, from the cancer cell line panel of the Broad-Novartis Cancer Cell Line Encyclopedia (CCLE). ${ }^{33}$ We focused on the three cancers with the most derived cell lines displaying WHSCIL1 and PPAPDC1B amplification. We selected several of these cell lines to evaluate the role of these two genes in cell survival in vitro, in anchorage-dependent and in anchorage-independent conditions, and in tumor growth in vivo with xenograft models.

\section{Materials and Methods}

Cell Lines

PANC08.13 and DMS114 (pancreatic and lung cancer cell lines, respectively) cells were purchased from ATCC (LGC standard, Molsheim, France); DANG and HUPT4 (pancreatic cancer cell lines) cells were purchased from the Cell Line Service (Eppelheim, Germany) and the German Resource Centre for Biological Material (DSMZ, Braunschweig, Germany), respectively.

\section{Cell Culture}

PANC08.13 cells were grown in RPMI 1640 medium with $15 \%$ fetal bovine serum, supplemented with $2 \mathrm{mmol} / \mathrm{L}$ L-glutamine and $10 \mathrm{U} / \mathrm{mL}$ human insulin, adjusted to give final concentrations of $1.5 \mathrm{~g} / \mathrm{L}$ sodium bicarbonate, $4.5 \mathrm{~g} / \mathrm{L}$ glucose, $10 \mathrm{mmol} / \mathrm{L}$ HEPES, and $1.0 \mathrm{mmol} / \mathrm{L}$ sodium pyruvate. DANG cells were grown in RPMI 1640 medium with $10 \%$ fetal bovine serum, supplemented with $2 \mathrm{mmol} / \mathrm{L} \mathrm{L-}$ glutamine. HUPT4 cells were grown in $80 \%$ modified Eagle's medium (with Earle's salts), with $20 \%$ fetal bovine serum, $1 \%$ nonessential amino acids, and $1 \%$ sodium pyruvate. DMS114 cells were grown in Waymouth's MB 752/1 medium with $10 \%$ fetal bovine serum. All reagents were purchased from Life Technologies (Saint-Aubin, France).

\section{RNA Extraction from Cell Lines}

RNA was isolated from cell lines with RNeasy Mini kits (Qiagen, Courtaboeuf, France).

\section{RNA Interference}

Transient transfections were performed, using Lipofectamine RNAimax, according to the manufacturer's instructions (Invitrogen, Saint-Aubin, France), with $20 \mathrm{nmol} / \mathrm{L}$ siRNA. A negative control siRNA and siRNAs specific for PPAPDC1B and WHSC1L1 were purchased from Qiagen. For each gene, the siRNA was designed to knock down the expression of all known mRNA isoforms. The sequences of the sense-strand siRNA were as follows: PPAPDC1B-1, $5^{\prime}-$ GAUGUUUGUUAUUGCAUUUTT-3'; PPAPDC1B-2, 5'GCCGAUGUUUGUUAUUGCATT-3'; WHSC1L1-1, 5'GGGUAUCCAUCAUCAAUCATT-3'; and WHSC1L1-2, 5'-CGAGAGUAUAAGGUCAUATT-3' .

\section{Lentivirus-Mediated shRNA Knockdown of Gene Expression}

We knocked down the expression of human WHSC1L1 and PPAPDC1B genes in the DMS114 and DANG cell lines, using the Mission TRC human shRNA clone sets (pLKO.1puro; Sigma, Saint-Quentin Fallavier, France), as previously described for breast cancer. ${ }^{17}$ Cells expressing shRNA were selected on $2 \mu \mathrm{g} / \mathrm{mL}$ puromycin for 6 weeks for functional studies (cell proliferation and xenografts) and for 4 weeks after infection for RNA extraction.

\section{Real-Time Quantitative RT-PCR}

Reverse transcription was performed with $1 \mu \mathrm{g}$ of total RNA, with the High-Capacity cDNA reverse transcription 
kit (Applied Biosystems, Saint-Aubin, France), and PCR was performed in a Roche real-time thermal cycler with Roche Syber Green Master Mix (Roche, Meylan, France). The sequences of the primers used were as follows: PPADCIB, $5^{\prime}$-CCACTGTCTCTGATCTTCCTGG-3' (sense) and $5^{\prime}$-GGTAAAGACGCCATTCAGAGCC-3' (antisense); and WHSClL1, 5'-CAGACGTTTCTGATGTGCAGTCC- $3^{\prime}$ (sense) and 5'-CTCCAGGTGAAAGTGTTTGCAGC- $3^{\prime}$ (antisense).

\section{Proliferation Assay}

PANC08.13 $\left(2 \times 10^{5}\right)$, HUPT4 $\left(1.5 \times 10^{5}\right)$, DANG $(1.5 \times$ $\left.10^{5}\right)$, and DMS114 $\left(3 \times 10^{5}\right)$ cells were plated in a 6-well plate and transfected with $20 \mathrm{nmol} / \mathrm{L}$ siRNA. The cells were treated with trypsin for 72 hours (for DANG and DMS114) and 96 hours (for PANC08.13 and HUPT4) after transfection and stained with trypan blue, and viable cells were counted in triplicate with a Malassez hematocytometer (Roche, Meylan, France).

\section{Soft Agar Assay}

We added 20,000 siRNA-transfected cells (PANC08.13, HUPT4, and DANG) in the corresponding growth medium and $0.35 \%$ agar to triplicate wells of a 12 -well plate containing medium and $0.8 \%$ agar, 24 hours after transfection. The plates were incubated for 21 days, and colonies of at least $50 \mu \mathrm{m}$ in diameter were scored as positive, on examination under a phase-contrast microscope equipped with a measuring grid.

\section{Xenografts in Nude Mice}

Six-week-old female Swiss nu/nu mice were obtained from Charles River Laboratories (Saint Germain sur l'Arbresle, France) and reared in the animal facility of the Curie Institute in specified pathogen-free conditions. Animals were housed and cared for in accordance with the institutional guidelines of the French National Ethics Committee (Ministère de l'Agriculture et de la Pêche, Direction Départementale des Services Vétérinaires, Paris, France) under the supervision of investigators with the required accreditation. We injected $9 \times$ $10^{5}$ DMS114 and $2 \times 10^{6}$ DANG cells s.c. into the flank (dorsal region) of each mouse.

Tumor formation was monitored for up to 60 days, and tumor size was determined weekly with Vernier calipers: two perpendicular diameters were used to estimate tumor volume according to the formula, $a b^{2} / 2$, where $a$ is the largest and $b$ is the smallest diameter.

\section{Statistics}

For PPAPDC1B and WHSC1L1, mRNA level (Affymetrix or RNA sequencing signal) and DNA copy number [singlenucleotide polymorphism (SNP) array, normalized signal] were compared in cell lines and in tumors, by Pearson's correlation test (Affymetrix, Paris, France). The levels of mRNA for PPAPDC1B and WHSC1L1 in nonamplified cell lines were compared with those in cell lines with an unchanged 8p-DNA copy number, in Student's $t$-tests. We evaluated the results of the functional experiments with the Student's $t$-test and the Wilcoxon rank-sum test for in vitro and in vivo experiments, respectively.

\section{Analysis of Publicly Available Data}

Both gene-centered mRNA data (custom-made Agilent chips) and copy number data (Agilent comparative genomic hybridization arrays) were obtained for 112 squamous cell carcinomas from The Cancer Genome Atlas consortium ${ }^{34}$ (https://tcga-data.nci.nih.gov/tcga/dataAccessMatrix.htm? mode $=$ ApplyFilter \&diseaseType $=$ LUSC, last accessed March 5, 2013). Copy number data for 65 small-cell lung carcinomas (SCLCs; Affymetrix SNP 6.0 arrays, Paris, France) and normalized gene expression for 15 of these tumors (RNA sequencing) were obtained from Bashyam et al. ${ }^{35}$ Gene expression (Affymetrix u133 plus 2.0) and copy number data (Affymetrix SNP 6.0 arrays) for 883 cell lines were obtained from the CCLE. ${ }^{33}$

For the analysis of copy number data, we defined gene amplification as a normalized SNP array $\log 2$ copy number ratio of more than one with respect to normal DNA (more than four copies), and gain as a normalized SNP array $\log 2$ copy number ratio between 0.6 and 1 (between three and four copies).

\section{Results}

PPAPDC1B and WHSC1L1 on Chromosome 8p11-12 Are Amplified and Overexpressed in Several Pancreatic Adenocarcinoma, Breast, and Lung Tumor-Derived Cell Lines

We investigated the role of PPAPDClB and WHSCIL1 as driver genes and potential therapeutic targets for tumors with 8p11-12 amplification from sites other than the breast. We began by identifying cancer-derived cell lines presenting PPAPDCIB and WHSC1L1 amplification and overexpression. We made use of publicly available data concerning gene copy number obtained with Affymetrix SNP6 arrays for 883 cancer-derived cell lines, and data concerning gene expression were obtained with the Affymetrix U133plus2.0 DNA array for most of these cell lines (807/ 883) from the CCLE. ${ }^{33}$ Amplification of both PPAPDC1B and WHSC1L1 in the 8p11-12 region, as defined by a normalized SNP array $\log 2$ copy number ratio of more than one with respect to normal DNA (more than four copies), was detected in 26 cancer cell lines (Figure 1 and Supplemental Table S1). These cell lines included six derived from breast cancers $(11.5 \%$ of the 52 breast cancer cell lines studied: MDAMB134VI, CAL120, JIMT1, ZR75.1, CAMA1, and 


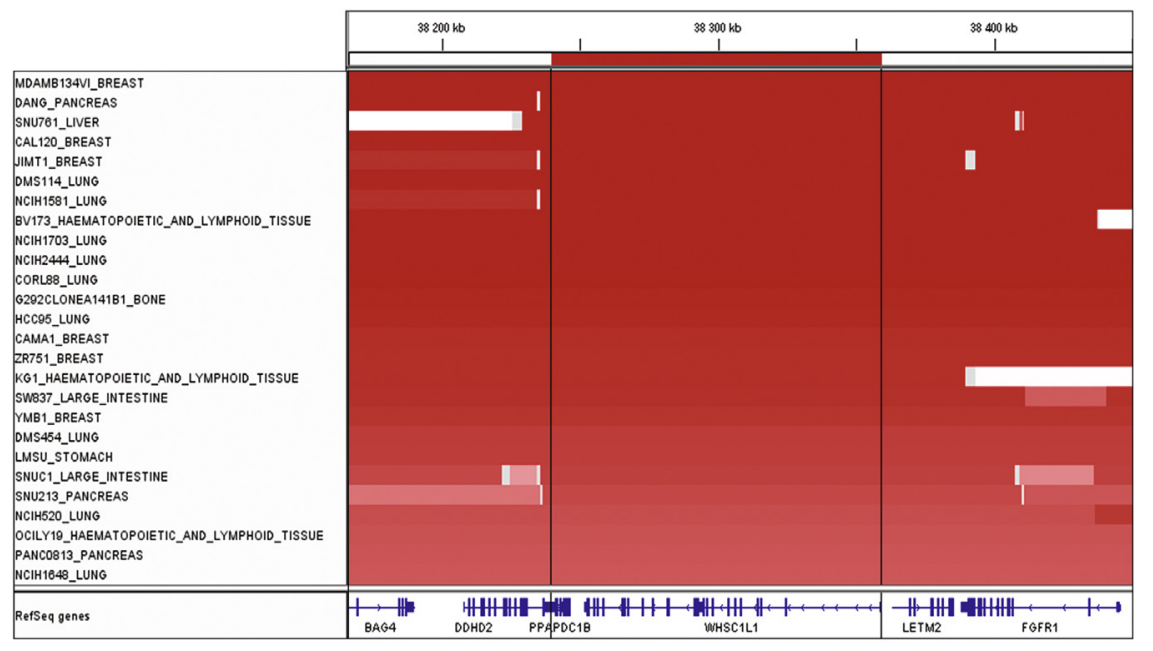

Figure 1 Amplification of the chromosome 8p11-12 region encompassing PPAPDC1B and WHSC1L1 in cancer cell lines. The Broad-Novartis Cancer Cell Line Encyclopedia (CCLE) ${ }^{33}$ contains gene copy number data obtained with Affymetrix SNP6 arrays for 883 cell lines derived from cancers from various primary sites. Cell lines were ordered by normalized SNP array log2 copy number ratio for the chromosome 8p11-12 region encompassing $P P A D C 1 B$ and WHSC1L1. Cell lines presenting an amplification of both PPAPDC1B and WHSC1L1 (with a normalized SNP array log2 copy number ratio of $>1.0$ ) are listed. The name and site of the primary cancer are indicated for each cell line.

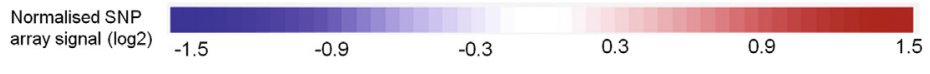

YMB1), three pancreatic cell lines (7.5\% of the 40 studied: DANG, SNU213, and PANC08.13), nine lung cancer cell lines $(5.6 \%$ of the 158 studied: DMS114, NCIH2444, NCIH1703, CORL88, NCIH1581, HCC95, DMS454, NCIH520, and NCIH1648), two large intestine cancer cell lines (3.9\% of the 51 studied: SW837 and SNUC1), three hematopoietic cancer cell lines (1.8\% of the 164 studied: BV173, KG1, and OCILY19), one liver cancer cell line (3.7\% of the 27 studied: SNU761), one stomach cancer cell line (2.7\% of the 37 studied: LSMU), and one bone cancer cell line (4\% of the 25 studied: G292CLONEA141B1).

We focused on cell lines derived from pancreatic and lung cancers, which were (after breast cancer, for which PPAPDCIB and WHSC1L1 have already been shown to act as driver genes) the two cancers with the most derived cell lines displaying PPAPDCIB and WHSCILI amplification, at 7.5\% and $5.6 \%$, respectively. Moreover, two other lung cell lines (Calu3 and NCIH1733) presented a gain, as shown by their normalized SNP array $\log 2$ copy number ratios, which were between 0.6 and 1 (more than three copies but less than four copies) (Supplemental Table S1). We found that, as in breast cell lines, there was a direct relationship between DNA copy number and expression level for both PPAPDC1B and WHSC1L1 in lung and pancreatic cell lines (Pearson's $r>0.6$, $P<0.0001)$, indicating that DNA copy number had a significant effect on gene expression (Figure 2A). Consistent with this finding, as in breast cancer-derived cell lines, $P P A P D C 1 B$ and $W H S C 1 L 1$ were significantly more strongly expressed in cell lines derived from lung and pancreatic cancers that displayed amplification than in cell lines from these sources without amplification (Student's $t$-test, $P<0.05$ ) (Figure 2B). We then investigated whether these correlations between gene amplification and gene overexpression were also relevant in the tumors from which the cell lines were derived. Pancreatic cell lines with PPAPDClB and WHSClL1 amplification were derived from pancreatic ductal adenocarcinomas, in which PPAPDC1B and WHSC1L1 have been shown to be gained in two different studies: in $10 \%$ of the tumors $(4 \text { of } 39)^{15}$ and in $7 \%$ of the tumors ( 1 of 13$){ }^{36}$ WHSC1L1 mRNA levels have been shown to be correlated with DNA copy number in pancreatic adenocarcinoma $(P=$ $0.039)$, but $P P A P D C 1 B$ expression was not evaluated in this study. ${ }^{36}$ To our knowledge, no public data were available to analyze correlation between PPAPDC1B expression and DNA copy number in pancreatic adenocarcinoma. No functional analysis of the amplified genes has been reported for the 8p1112 amplicon, the role of which in pancreatic cancers remains unknown. ${ }^{35,37}$ Lung cell lines presenting amplifications of PPAPDC $1 B$ and WHSCIL1 were derived from SCLCs (three of nine) or from non-small-cell lung carcinomas (NSCLCs) (six of nine). For SCLCs, Peifer et $\mathrm{al}^{38}$ obtained SNP data for 65 tumors and RNA-seq data for WHSC1L1 for 15 of these tumors. These data showed an amplification of $P P A P D C 1 B$ and WHSC1L1 in one tumor (1.5\%) and gains in seven tumors (10\%) (Figure 3A). They also showed a significant correlation between WHSClL1 expression and DNA copy number (Figure 3B). No RNA-seq data were obtained for PPAPDC1B in this study. ${ }^{38}$ For NSCLCs, $14 \%$ of the tumors ( 6 of 44 ) were shown to present WHSCILI and PPAPDCIB amplification; WHSC1L1 amplification was linked to the overexpression of this gene, whereas the level of expression of PPAPDC1B was not determined. ${ }^{13}$ By using data from The Cancer Genome Atlas, ${ }^{34}$ we found that PPADC1B and WHSClL1 were amplified in 7\% (8 of 112) and gained in 10\% (12 of 112) of the squamous cell carcinomas considered (Figure 3C). Furthermore, the levels of mRNA for both PPAPDC1B and WHSClL1 were significantly correlated with DNA copy number in this subtype of NSCLCs (Figure 3D). WHSC1L1 has already been implicated in NSCLCs (in an adenocarcinoma-derived cell line), ${ }^{13}$ but the role of 

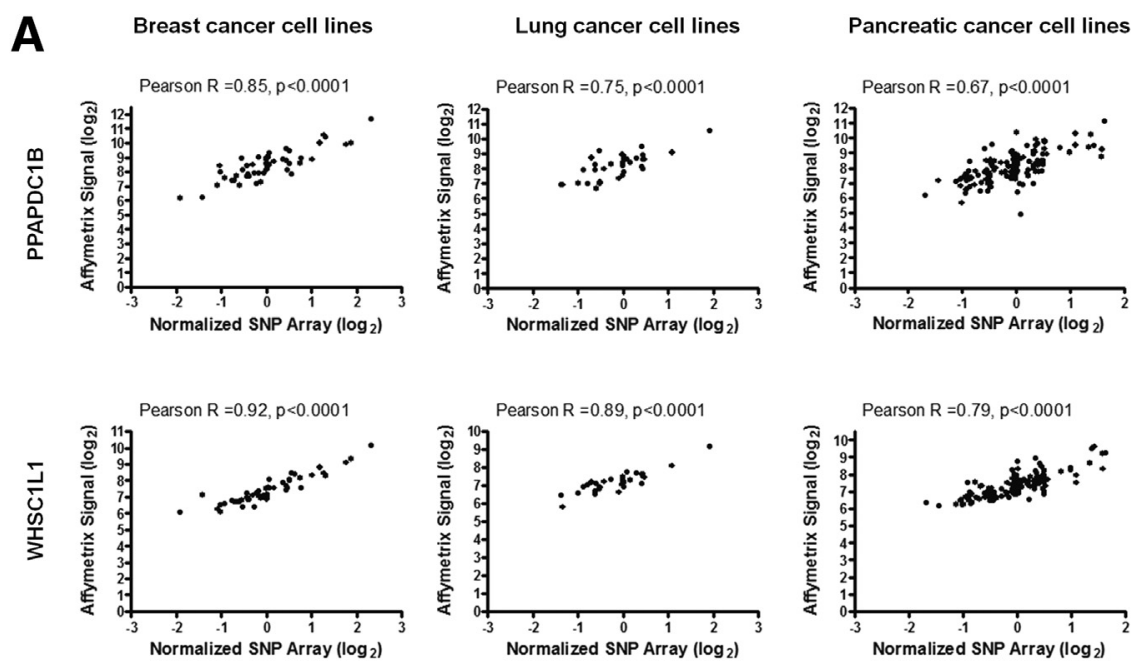

Figure 2 Amplification and overexpression of PPAPDC1B and WHSC1L1 in lung and pancreatic cancer cell lines. A: Correlation analysis of the 51 breast cancer cell lines, 151 lung cancer cell lines, and 33 pancreatic cancer cell lines for which both DNA copy number (SNP6 array) and gene expression data (Affymetrix U133plus2.0 array) were available from the Broad-Novartis Cancer Cell Line Encyclopedia (CCLE). ${ }^{33}$ RNA levels and copy number ratio were compared, in Pearson's correlation test. B: A Student's $t$-test comparison of gene expression levels between cell lines presenting PPAPDC1B and WHSC1L1 amplification and cell lines without amplification. ${ }^{*} P<0.05$, $* * * * P<0.0001$

PPAPDC1B in lung cancer (SCLC or NSCLC) has not been investigated before.

\section{Both WHSC1L1 and PPAPDC1B Are Drivers of the 8p11-12 Amplicon in Pancreatic Ductal Adenocarcinoma and Small-Cell Lung Cancer}

Based on our results, we selected four cell lines for further cell-based functional studies of PPAPDCIB and WHSC1L1: two pancreatic ductal adenocarcinoma-derived cell lines (DANG and PANC08.13) and one SCLC-derived cell line (DMS114), presenting amplification and overexpression of $P P A P D C 1 B$ and WHSC1L1, and one nonamplified cell line with low levels of WHSC1L1 and PPAPDC1B expression, the pancreatic cancer-derived cell line, HUPT4 (Figures 1 and 2B and Supplemental Table S1). Quantitative RTPCR (RT-qPCR) experiments confirmed that both genes were less strongly expressed in HUPT4 cells than in the cell lines with amplification, DANG, PANC08.13, and DMS114 (by a factor of 20) (Figure 4A).

RNA interference technology was then used for loss-offunction analysis and investigation of the role of PPAPDC1B and WHSC1L1 in the proliferation and survival of lung and pancreatic cancer cells. Two siRNAs per gene and a negative
siRNA control were used for each cell line. By using RTqPCR, we verified that 48 hours after transfection, PPAPDC1B and WHSC1L1 knockdowns were effective in all of the cell lines used (Figure 4B). The silencing of one gene had no effect on the level of expression of the other gene (data not shown). The specific knockdown of WHSCIL1 yielded fewer viable lung (DMS114) and pancreatic (DANG and PANC08.13) cancer cells than transfection with the control siRNA (55\% to $70 \%$ inhibition, depending on the cell line and siRNA used) (Figure 4C). The specific knockdown of PPAPDC1B also decreased the cell viability of the lung cancer cell line (DMS114; 55\% to 60\% inhibition) and that of one of the two pancreatic cancer cell lines presenting PPAPDC1B amplification and overexpression (DANG; 40\% inhibition) (Figure 4C).

Similar ranges of decrease in cell survival were observed with both siRNAs for each gene, consistent with specific, rather than off-target, effects. Furthermore, none of the siRNAs affected HUPT4 cell count (cells expressing both PPAPDC1B and WHSCIL1 at much lower levels than the cell lines displaying amplification) (Figure 4A). This observation was also consistent with specific, rather than off-target, effects of siRNAs in the other cell lines and excluded the possibility of WHSCIL1 and $P P A P D C 1 B$ being essential genes (genes involved in the survival of every cell regardless of their expression level), 

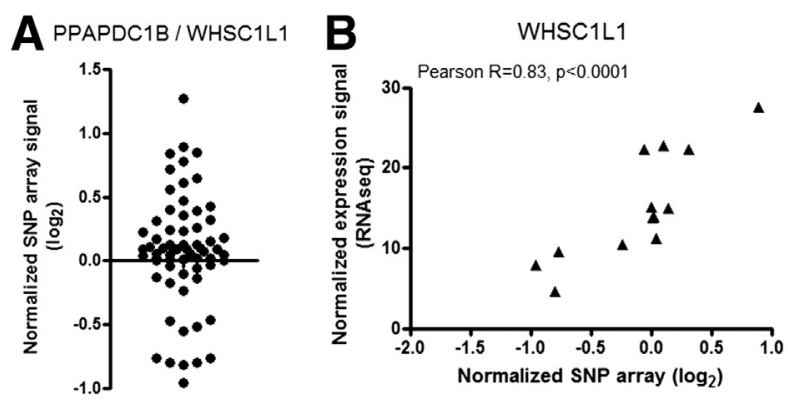

D

PPAPDC1B

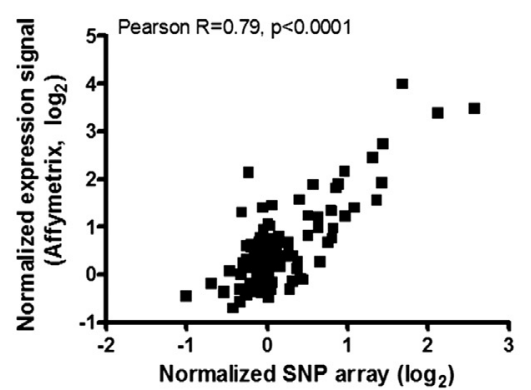

C $_{\text {PPAPDC1B / WHSC1L1 }}$

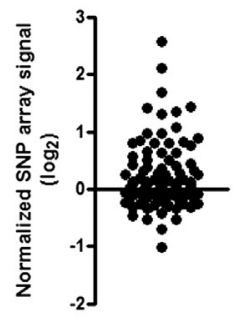

WHSC1L1

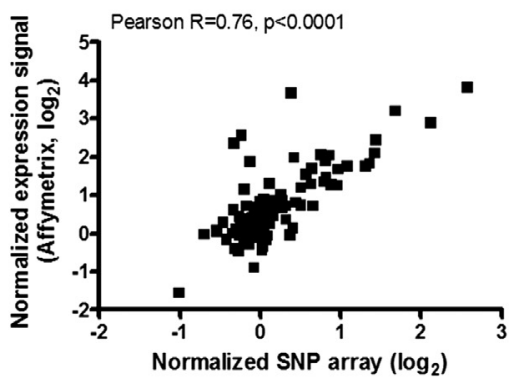

Figure 3 Amplification and overexpression of PPAPDC1B and WHSC1L1 in lung cancer. A: Analysis of PPAPDC1B and WHSC1L1 copy number in smallcell lung tumors $(n=65)$. SNP data were available from Peifer et al. ${ }^{38}$ B: Correlation of WHSC1L1 expression (RNA-seq data) and copy number (SNP data) for 15 small-cell lung tumors for which both types of data were available. ${ }^{38} \mathbf{C}$ and $\mathbf{D}$ : Analysis of PPAPDC1B and WHSC1L1 copy number in lung squamous carcinoma, a subtype of NSCLC $(n=$ 112). C: Correlation of WHSC1L1 and PPAPDC1B expression levels (Affymetrix signal) with copy number (SNP data) in lung squamous carcinoma $(n=112)$. Data were obtained from The Cancer Genome Consortium. ${ }^{34}$ suggesting instead that the observed effects resulted directly from the overexpression of these genes (Figure 4C).

Anchorage-independent growth is a hallmark of oncogenic transformation. We, therefore, investigated the role of PPAPDC1B and WHSC1L1 in the survival/proliferation of clonogenic cells in soft agar assays. We demonstrated that WHSCIL1 silencing significantly decreased, by $45 \%$ to $55 \%$, the ability of both pancreatic cancer cell lines presenting an 8p11-12 amplification (DANG and PANC08.13) to form colonies on soft agar, whereas PPAPDC1B knockdown inhibited only DANG colony formation (35\% to $45 \%$ inhibition) (Figure 4D). Under described growth conditions, the DMS114 lung cancer cell line was unable to form colonies in the soft agar assay (data not shown).

Taken together, these results suggest that both PPAPDC1B and $W H S C 1 L 1$ regulate cell viability in lung cancers with $8 \mathrm{p}$ amplification. WHSCILI also seems to regulate the cell viability (in anchorage-dependent and anchorage-independent conditions) of pancreatic cancer cells with $8 \mathrm{p}$ amplification, whereas the role of PPAPDC1B seems to depend on the pancreatic cancer cells considered. Furthermore, as previously reported for breast cancer cell lines with 8p11-12 amplification, ${ }^{17}$ the simultaneous inhibition of both of these genes had no additive or synergic effect on cell viability in either pancreatic or lung cancer-derived cell lines (Figure 4E).

Both WHSC1L1 and PPAPDC1B Regulate the Growth of Pancreatic Ductal Adenocarcinoma and Small-Cell Lung Cancer-Derived Cell Tumors with 8p11-12 Amplification in Vivo in Xenograft Models

We investigated the role of PPAPDC1B and WHSC1L1 in the growth in vivo of tumors formed from SCLC- and pancreatic carcinoma-derived cells, by establishing stable cell lines with PPAPDC1B or WHSC1L1 knockdown. This long-term knockdown of the expression of these genes after the stable expression of shRNAs in the DMS114 and DANG cell lines fully confirmed the effects observed with siRNAs (Figure 5, A and B). Indeed, both WHSC1L1 and PPAPDC1B shRNAs efficiently knocked down WHSC1L1 and PPAPDC1B transcript levels, by $80 \%$ to $90 \%$ with respect to the control shRNA (Figure $5 \mathrm{~A}$ ). Cells expressing WHSC1L1 or PPAPDC1B shRNAs grew much more slowly than control cells, resulting in a threefold to fourfold difference in the number of shRNA-expressing cells 10 days after seeding (Figure 5B).

For each gene and cell line, one shRNA-expressing stable cell line (the fastest-growing cell line) was amplified and implanted s.c. in athymic nude mice (DANG: PPAPDC1B shRNA558, WHSCILI shRNA1959, and control shRNA; DMS114: PPAPDC1B shRNA242, WHSC1L1 shRNA738, and control shRNA). PPAPDCIB and WHSCIL1 long-term knockdown inhibited the tumor growth of xenografts of adenocarcinoma-derived cells (DANG) and SCLC-derived cells (DMS114) (Figure 5C). Thirty-eight days after the injection of DANG cells, mice that received PPAPDCIB shRNA- or WHSC1L1 shRNA-expressing cells were found to have developed significantly fewer tumors than those receiving control shRNA-expressing cells (two of eight and three of eight, respectively, versus eight of eight; Fisher's exact test; $P=0.007$ for WHSClL1 and $P=0.025$ for PPAPDC1B) (data not shown). Tumor volume was $90 \%$ lower in mice receiving PPAPDC1B or WHSC1L1 shRNA-expressing cells than in mice receiving control shRNA-expressing DANG cells (Wilcoxon rank-sum test, $P<0.001$ ) (Figure 5C). Fifty-eight days after the injection of DMS114 cells, mice receiving 

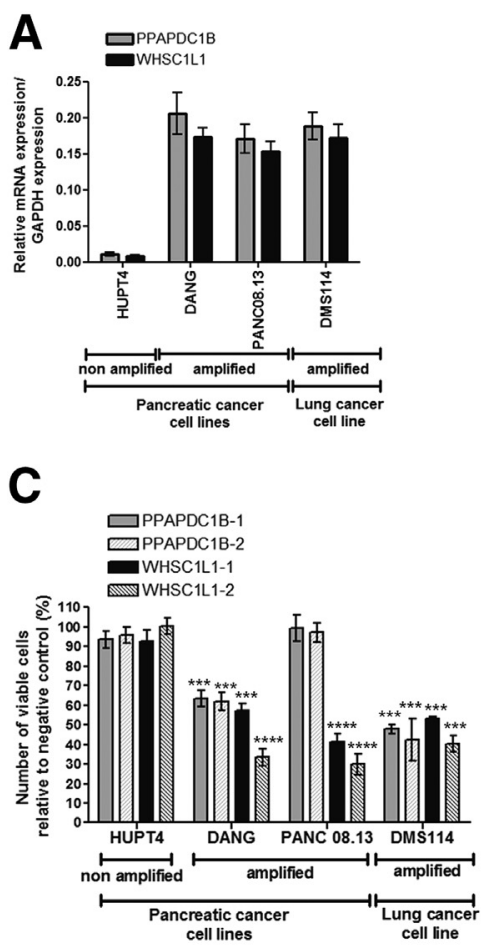

B
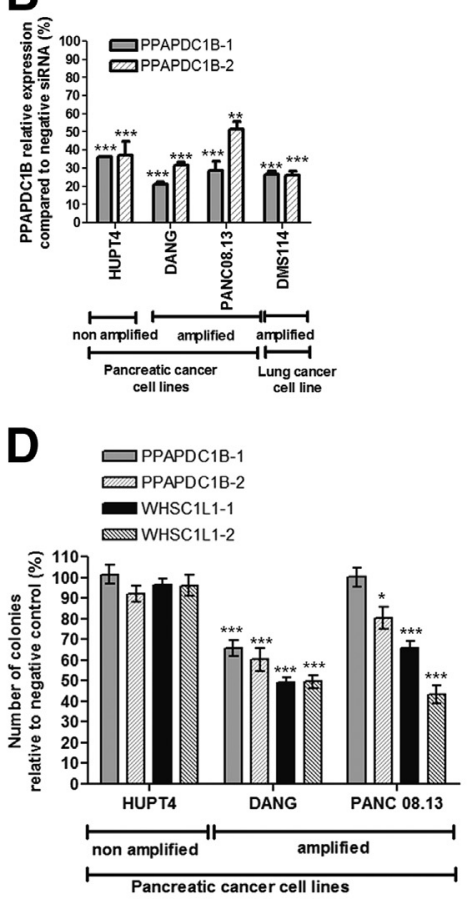

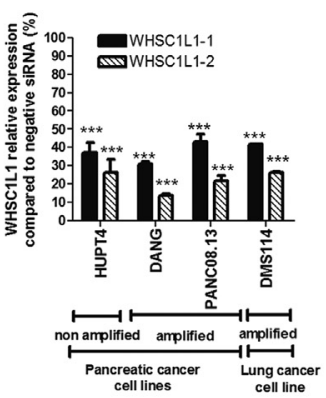

$\mathbf{E}$

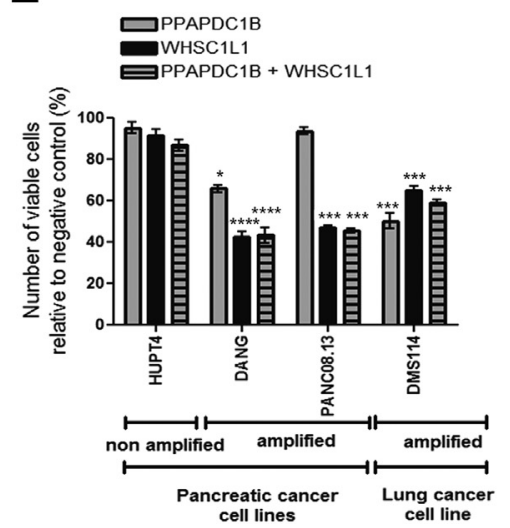

Figure 4 PPAPDC1B and WHSC1L1 regulate the viability of pancreatic adenocarcinoma- and SCLC-derived cells with 8p11-12 amplification in vitro. A: Levels of mRNA for PPAPDC1B and WHSC1L1, normalized with respect to GAPDH, as assessed by RT-qPCR. B: Efficiency of mRNA silencing by siRNAs specific for PPAPDC1B or WHSC1L1. The level of RNA for each gene was assessed and expressed relative to that for GAPDH, 48 hours after transfection with the specific or control siRNA. C and D: Effect of PPAPDC1B and WHSC1L1 knockdown on cell viability in anchorage-dependent conditions (C) and anchorage-independent conditions (colony formation in soft agar) (D). E: Effect of PPAPDC1B and WHSC1L1 knockdowns on cell viability. B-E: Results are expressed with respect to the control siRNA, which had no effect on cell behavior, as shown by comparison with the transfection reagent alone (data not shown). Results are the means $\pm \mathrm{SD}$ of three independent experiments performed in triplicate. Results were compared in Student's $t$-tests. ${ }^{*} P<0.05,{ }^{* * *} P<0.001$, and ${ }^{* * * * P}<0.0001$.

PPAPDCIB shRNA- or WHSCIL1 shRNA-expressing cells were found to have developed significantly fewer tumors than those receiving control shRNA-expressing cells (one of eight for WHSC1L1 or PPAPDC1B shRNA versus seven of eight for control shRNA; Fisher's exact test; $P=0.01$ ) (data not shown). Consistent with these findings, tumor volume was significantly lower in mice receiving cells expressing specific shRNA than in mice receiving cells expressing the control shRNA (Figure 5C).

The Genes Targeted by PPAPDC1B and WHSC1L1 in Cell Lines Derived from Lung and Pancreatic Cancers Differ from Those Targeted in Breast Cancer

Because PPAPDC1B and WHSC1L1 knockdowns had inhibitory effects in cell lines derived from lung and pancreatic cancers with 8p11-12 amplification that were similar to those in breast cancer-derived cell lines, we looked for similarities in the underlying mechanisms.

The shRNA knockdown of PPAPDC1B in the ZR75.1 breast cancer cell line displaying 8p11-12 amplification showed several genes to be regulated by the product of this gene and to be coordinately regulated in tumors with amplification, with respect to tumors without amplification and normal samples. These regulated genes included DUSP22, which is thought to affect PPAPDC1B activity by regulating the estrogen receptor pathway, and IL6ST, PRL, and $S T C 1$, which may mediate signal transduction, via the Jak-STAT signaling pathway in particular. ${ }^{17}$ By using RTqPCR, we demonstrated that PPAPDC1B knockdown in DANG (pancreatic) and DMS114 (lung) cancer cell lines had no effect on the levels of expression of these four genes, suggesting that the genes regulated by PPAPDC1B amplification/overexpression may be cell type specific (data not shown).

The genes targeted by the product of the WHSCILI gene in breast cancers have also been identified, by comparing gene expression levels in MCF10A-WHSC1L1 cells and MCF10A breast cells. Thirty-six of these genes were found to be coordinately regulated in SUM-44 breast cancer cell lines with 8p11-12 amplification, with respect to the MCF10A cell line (without 8p11-12 amplification). ${ }^{39}$ Among these genes, three were of particular interest because of their possible involvement in a transcriptional network regulating WNT signaling: IRX3 (a member of the homeobox family), TBLXI, and SFRP $1 .{ }^{39}$ The RT-qPCR analysis showed that these three genes, such as RAGIAP (one of the genes displaying the highest degree of overexpression in MCF10-WHSC1L1 cells 
A

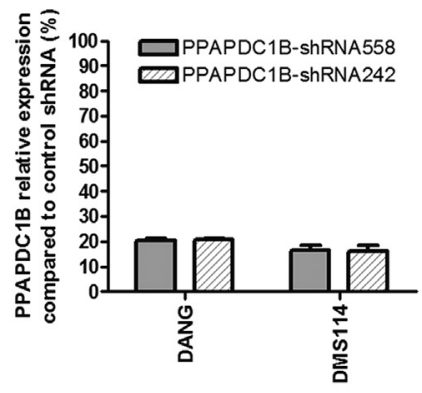

C

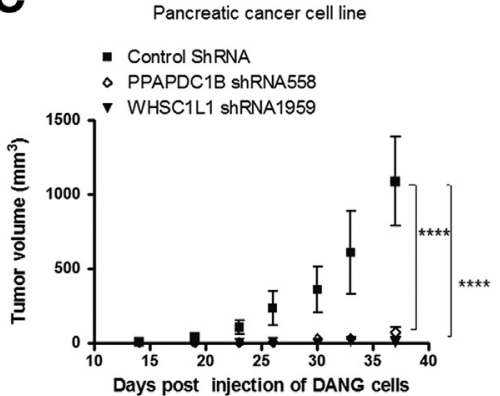

B
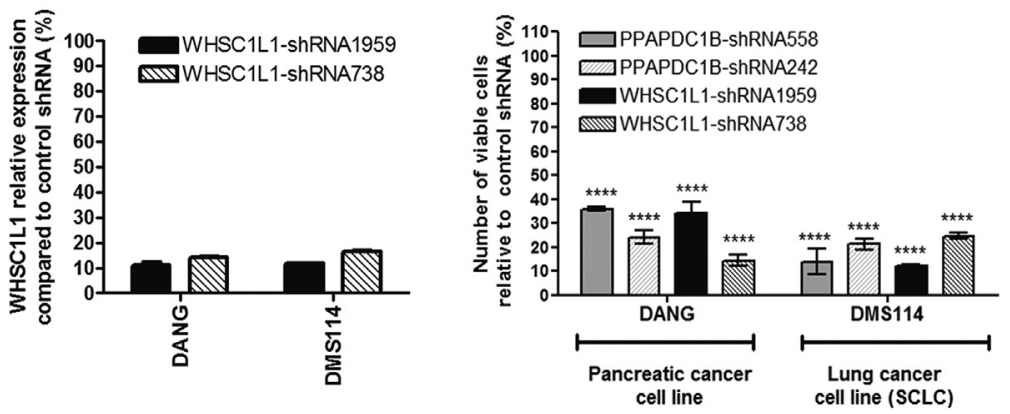

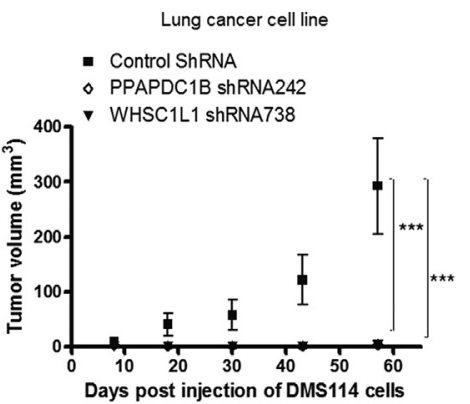

Figure 5 In vitro and in vivo effects of long-term PPAPDC1B and WHSC1L1 knockdown with shRNA. A: Efficiency of PPADC1B and WHSC1L1 mRNA silencing by the specific shRNAs. The level of expression of each gene with respect to that of TBP was assessed 4 weeks after infection with a lentivirus encoding shRNA and the selection of infected cells on puromycin. The results shown are from two independent experiments. B: Effect of gene knockdown on cell growth. Results are expressed with respect to the control shRNA. Results are the means \pm SD of two experiments performed in triplicate. A Student's $t$-test was used. C: Pancreatic adenocarcinoma-derived cells (DANG) or SCLC-derived cells (DMS114) expressing PPAPDC1B shRNA, WHSC1L1 shRNA, or control shRNA were injected s.c. into nude mice ( $n=8$ per group). The growth of the xenografts was followed weekly. Results are the means \pm SEM and were compared in Wilcoxon's rank-sum test. ${ }^{* *} P<0.001,{ }^{* * * * P}<0.0001$.

with respect to MCF10 cells), were not WHSC1L1 targets in the cell lines derived from lung and pancreatic cancers with 8p11-12 amplification (data not shown).

\section{Discussion}

With publicly available large-scale data (transcriptome and genomic alterations) for many cancers, it is possible to search for candidate oncogenes involved in several cancers, by searching for genes that are overexpressed when amplified in different tumor cell types. By using the available data from the cancer cell line panel of the CCLE, ${ }^{33}$ we identified pancreatic adenocarcinoma, lung (NSCLC and SCLC), and breast cancer-derived cell lines presenting amplification and overexpression of two candidate driver oncogenes, $P P A P D C 1 B$ and WHSC1L1, within the 8p11-12 chromosomal region. WHSC1L1 played an oncogenic role in all of the cell lines presenting amplification that we tested, whereas PPAPDC1B knockdown did not alter cell viability and/or colony-forming ability in one of the three cell lines presenting 8p11-12 amplification studied. The oncogenic role of WHSC1L1 had already been demonstrated in non-small-cell lung cancerand breast cancer-derived cell lines. ${ }^{13,17}$ We showed an additional oncogenic role in pancreatic adenocarcinomaand small-cell lung cancer-derived cell lines. PPAPDC1B had previously been demonstrated to be oncogenic only in breast cancer. ${ }^{17}$ We showed that it is also oncogenic in some SCLC- and pancreatic adenocarcinoma-derived cell lines presenting amplifications of the 8p11-12 region. It was long thought that each individual amplified region contained only one driver oncogene. However, several examples are known of the presence of various oncogenes in a single amplicon. Nevertheless, the driver oncogenes within a given amplicon may not be the same in different types of cancer. Indeed, FGF19 was recently shown to act, with cyclin D1, as a driver gene for the chromosome 11q13.3 amplicon in hepatocellular carcinoma but to have no oncogenic role in breast carcinomas with 11q13.3 amplification. ${ }^{40}$ One of the first examples of multiple driver genes to be described was that of the $17 \mathrm{q} 12$ amplicon (alias ERBB2 amplicon). This amplicon is the most frequently found amplicon in breast cancers; it contains two neighboring genes, STARD3 and GRB7, in addition to $E R B B 2$, that have been shown to be oncogenic when amplified. ${ }^{41}$ We and others have demonstrated the presence of numerous oncogenes, including C8orf4, LSM1, BAG4, $P P A P D C 1 B$, and WHSC1L1, 12,17,22 and, more recently, ZNF703, ${ }^{18,19}$ in the 8p11-12 amplicon in breast cancers. These oncogenes may also be involved in other cancers presenting the 8p11-12 amplicon. We focused herein on two genes located in the 8p11-12 amplicon that had previously been described as oncogenes in breast cancer and encoded 
potentially druggable enzymes, WHSC1L1 and PPAPDCIB. We showed that both these genes were also oncogenic driver genes in pancreatic adenocarcinoma and lung cancer. We did not study the driver gene role of the other amplified genes within the 8p11-12 amplicon in pancreatic adenocarcinoma and lung cancer. The minimal region of amplification in the lung cancer cell lines studied by the CCLE encompassed 12 genes $(37,750$ to $38,450 \mathrm{~kb}: A D R B 3, E I F 4 E B P 1, A S H 2 L$, STAR, LSM1, BAG4, DDHD2, PPAPDC1B, WHSC1L1, LETM2, FGFR1, and C8ORF86), whereas that studied in pancreatic cancer cell lines encompassed 21 genes $(37,000$ to 38,700 kb: ZNF703, ERLIN2, PROSC, GPR124, BRF2, RAB11FIP1, GOTL1, ADRB3, EIF4EBP1, ASH2L, STAR, LSM1, BAG4, DDHD2, PPAPDC1B, WHSC1L1, LETM2, FGFR1, C8ORF86, RNF5P, and TACC1; data not shown). It is highly probable that, as in breast cancers, the $8 \mathrm{p} 11-12$ amplicon in pancreatic adenocarcinoma and lung cancers contains other oncogenic driver genes in addition to $P P A P D C 1 B$ and WHSC1L1, which we show herein to be driver genes in these cancers. PPAPDCIB and WHSCILI may also play an oncogenic role in other cancers, such as bladder cancers, in which we observed the overexpression of WHSC1L1 and PPAPDC1B in tumors with the 8p11-12 amplicon (data not shown). However, we did not perform functional studies on this cancer, because no bladder cancer cell line presenting this amplicon has yet been described. PPAPDC1B and WHSC1L1 amplifications were also identified in two large intestinal cancer cell lines (SNUC1 and SW837) that did not present amplification of the other two main oncogenes of the 8p11-12 amplicon (FGFRI and ZNF703) (Figure 1 and data not shown). PPAPDC1B and WHSCIL1 are, therefore, probably driver genes of the 8p1112 amplicon in these cell lines. For any given region, the genes found to play an oncogenic role when amplified in one cell line may not necessarily prove oncogenic in all cell lines in which they are amplified. Indeed, we found that $P P A P D C 1 B$ was overexpressed and amplified in two pancreatic cell lines, DANG and PANC08.13, but that this gene was oncogenic only in the DANG cell line. Similar findings have previously been reported for FGFRl, which is not oncogenic in all breast cell lines in which it is amplified. ${ }^{42,43}$ These differences between cell lines may be the result of other alterations (mutations or amplifications) to genes encoding proteins involved in the oncogene transduction pathway in cell lines in which oncogene knockdown had no effect on cell transformation.

In cell lines in which both PPAPDC1B and WHSC1L1 were oncogenic, the inactivation of both genes revealed an absence of synergic or additive effects, suggesting that the products of these genes may activate the same, as yet poorly characterized, transduction pathways. We might expect the transduction pathways activated by these two genes to be common to the various cancers in which these genes display oncogenic activity. However, our results suggest that the targeted genes are cancer specific, because none of the nine breast cancer target genes tested was also found to be a target in lung or pancreatic cancer.
Several of the genes acting as oncogenes when amplified are potential treatment targets, because they are enzymes that could be targeted with small molecules blocking the catalytic site or have an extracellular domain that could be targeted with specific antibodies recognizing the extracellular part of the molecule. WHSC1L1 and PPAPDC1B are both enzymes, and PPAPDC1B has an extracellular domain. WHSC1L1 is a histone methyl transferase and is, therefore, involved in epigenetic mechanisms of gene regulation. The drugs available for targeting epigenetic mechanisms in clinical practice are toxic and not specific. Targeted treatments, focusing on WHSC1L1, in particular, are, therefore, required. It has been known for some time that kinases can behave as oncogenes. Phosphatases, such as PTP1B ${ }^{31,44-46}$ and PPAPDC1B,,${ }^{174}$ have also been shown more recently to play an oncogenic role in breast cancer. These findings, and data implicating phosphatases in other diseases, have led to interest in the development of drugs active against phosphatases. This demonstration of the involvement of WHSC1L1 and PPAPDC1B in several cancers in which they are amplified and overexpressed in $7 \%$ to $15 \%$ of patients, depending on the cancer concerned, highlights the importance of pan/trans-cancer studies and the power of publicly available large-scale genomic/transcriptomic data for such studies. Furthermore, it should trigger the development of drugs targeting these two enzymes. Such an approach may be particularly valuable for pancreatic cancer, a deadly cancer for which few therapeutic options are available. Furthermore, the potentially simultaneous action of the two enzymes in tumors with 8p11-12 amplification opens up new possibilities for combined treatments, making it possible to limit drug resistance.

We focused on cell lines with an 8p11-12 amplicon. Several mechanisms, in addition to genomic amplification, may account for the involvement of a given gene in cancers. For example, epidermal growth factor receptor (EGFR) amplification is observed in several cancers, but cetuximab, a treatment targeting EGFR and already in clinical use, has proved effective for the treatment of colorectal cancer, in which no genetic alteration of EGFR is observed. ${ }^{47}$ Thus, it may be useful to extend the potential of treatments targeting PPAPDC1B and WHSC1L1 to assess the role of these two proteins in cancers that overproduce this protein but do not necessarily have an 8p11-12 amplicon.

\section{Supplemental Data}

Supplemental material for this article can be found at http://dx.doi.org/10.1016/j.ajpath.2013.07.028.

\section{References}

1. Albertson DG: Gene amplification in cancer. Trends Genet 2006, 22: 447-455

2. Albertson DG, Collins C, McCormick F, Gray JW: Chromosome aberrations in solid tumors. Nat Genet 2003, 34:369-376 
3. Arteaga CL, Moulder SL, Yakes FM: HER (erbB) tyrosine kinase inhibitors in the treatment of breast cancer. Semin Oncol 2002, 29: $4-10$

4. Badache A, Goncalves A: The ErbB2 signaling network as a target for breast cancer therapy. J Mammary Gland Biol Neoplasia 2006, 11: $13-25$

5. de Bono JS, Rowinsky EK: The ErbB receptor family: a therapeutic target for cancer. Trends Mol Med 2002, 8:S19-S26

6. Birnbaum D, Adelaide J, Popovici C, Charafe-Jauffret E, Mozziconacci MJ, Chaffanet M: Chromosome arm 8p and cancer: a fragile hypothesis. Lancet Oncol 2003, 4:639-642

7. Adelaide J, Chaffanet M, Imbert A, Allione F, Geneix J, Popovici C, van Alewijk D, Trapman J, Zeillinger R, Borresen-Dale AL, Lidereau R, Birnbaum D, Pebusque MJ: Chromosome region 8p11p21: refined mapping and molecular alterations in breast cancer. Genes Chromosomes Cancer 1998, 22:186-199

8. Garcia MJ, Pole JC, Chin SF, Teschendorff A, Naderi A, Ozdag H, Vias M, Kranjac T, Subkhankulova T, Paish C, Ellis I, Brenton JD, Edwards PA, Caldas C: A $1 \mathrm{Mb}$ minimal amplicon at 8p11-12 in breast cancer identifies new candidate oncogenes. Oncogene 2005, 24: $5235-5245$

9. Gelsi-Boyer V, Orsetti B, Cervera N, Finetti P, Sircoulomb F, Rouge C, Lasorsa L, Letessier A, Ginestier C, Monville F, Esteyries S, Adelaide J, Esterni B, Henry C, Ethier SP, Bibeau F, Mozziconacci MJ, CharafeJauffret E, Jacquemier J, Bertucci F, Birnbaum D, Theillet C, Chaffanet M: Comprehensive profiling of 8p11-12 amplification in breast cancer. Mol Cancer Res 2005, 3:655-667

10. Theillet C, Adelaide J, Louason G, Bonnet-Dorion F, Jacquemier J, Adnane J, Longy M, Katsaros D, Sismondi P, Gaudray P, Birnbaum D: FGFRI and PLAT genes and DNA amplification at 8p12 in breast and ovarian cancers. Genes Chromosomes Cancer 1993, 7:219-226

11. Ugolini F, Adélaïde J, Charafe-Jauffret E, Nguyen C, Jacquemier J, Jordan B, Birnbaum D, Pébusque MJ: Differential expression assay of chromosome arm 8p genes identifies Frizzled-related (FRP1/FRZB) and fibroblast growth factor receptor 1 (FGFR1) as candidate breast cancer genes. Oncogene 1999, 18:1903-1910

12. Yang ZQ, Streicher KL, Ray ME, Abrams J, Ethier SP: Multiple interacting oncogenes on the 8p11-p12 amplicon in human breast cancer. Cancer Res 2006, 66:11632-11643

13. Tonon G, Wong KK, Maulik G, Brennan C, Feng B, Zhang Y, Khatry DB, Protopopov A, You MJ, Aguirre AJ, Martin ES, Yang Z, Ji H, Chin L, Depinho RA: High-resolution genomic profiles of human lung cancer. Proc Natl Acad Sci U S A 2005, 102:9625-9630

14. Williams SV, Platt FM, Hurst CD, Aveyard JS, Taylor CF, Pole JC, Garcia MJ, Knowles MA: High-resolution analysis of genomic alteration on chromosome arm $8 \mathrm{p}$ in urothelial carcinoma. Genes Chromosomes Cancer 2010, 49:642-659

15. Birnbaum DJ, Adélaïde J, Mamessier E, Finetti P, Lagarde A, Monges G, Viret F, Goncalvès A, Turrini O, Delpero JR, Iovanna J, Giovannini M, Birnbaum D, Chaffanet M: Genome profiling of pancreatic adenocarcinoma. Genes Chromosomes Cancer 2011, 50: 456-465

16. Cooke SL, Pole JC, Chin SF, Ellis IO, Caldas C, Edwards PA: Highresolution array CGH clarifies events occurring on $8 \mathrm{p}$ in carcinogenesis. BMC Cancer 2008, 8:288

17. Bernard-Pierrot I, Gruel N, Stransky N, Vincent-Salomon A, Reyal F, Raynal V, Vallot C, Pierron G, Radvanyi F, Delattre O: Characterization of the recurrent 8p11-12 amplicon identifies PPAPDC1B, a phosphatase protein, as a new therapeutic target in breast cancer. Cancer Res 2008, 68:7165-7175

18. Holland DG, Burleigh A, Git A, Goldgraben MA, Perez-Mancera PA, Chin SF, Hurtado A, Bruna A, Ali HR, Greenwood W, Dunning MJ, Samarajiwa S, Menon S, Rueda OM, Lynch AG, McKinney S, Ellis IO, Eaves CJ, Carroll JS, Curtis C, Aparicio S, Caldas C: ZNF703 is a common Luminal B breast cancer oncogene that differentially regulates luminal and basal progenitors in human mammary epithelium. EMBO Mol Med 2011, 3:167-180
19. Sircoulomb F, Nicolas N, Ferrari A, Finetti P, Bekhouche I, Rousselet E, Lonigro A, Adelaide J, Baudelet E, Esteyries S, Wicinski J, Audebert S, Charafe-Jauffret E, Jacquemier J, Lopez M, Borg JP, Sotiriou C, Popovici C, Bertucci F, Birnbaum D, Chaffanet M, Ginestier C: ZNF703 gene amplification at 8p12 specifies luminal B breast cancer. EMBO Mol Med 2011, 3: $153-166$

20. Streicher KL, Yang ZQ, Draghici S, Ethier SP: Transforming function of the LSM1 oncogene in human breast cancers with the 8p11-12 amplicon. Oncogene 2007, 26:2104-2114

21. Turner N, Pearson A, Sharpe R, Lambros M, Geyer F, LopezGarcia MA, Natrajan R, Marchio C, Iorns E, Mackay A, Gillett C, Grigoriadis A, Tutt A, Reis-Filho JS, Ashworth A: FGFR1 amplification drives endocrine therapy resistance and is a therapeutic target in breast cancer. Cancer Res 2010, 70:2085-2094

22. Yang ZQ, Liu G, Bollig-Fischer A, Giroux CN, Ethier SP: Transforming properties of 8p11-12 amplified genes in human breast cancer. Cancer Res 2010, 70:8487-8497

23. Nakao K, Mehta KR, Fridlyand J, Moore DH, Jain AN, Lafuente A, Wiencke JW, Terdiman JP, Waldman FM: High-resolution analysis of DNA copy number alterations in colorectal cancer by array-based comparative genomic hybridization. Carcinogenesis 2004, 25: $1345-1357$

24. Tomlinson DC, Lamont FR, Shnyder SD, Knowles MA: Fibroblast growth factor receptor 1 promotes proliferation and survival via activation of the mitogen-activated protein kinase pathway in bladder cancer. Cancer Res 2009, 69:4613-4620

25. Veltman JA, Fridlyand J, Pejavar S, Olshen AB, Korkola JE, DeVries S, Carroll P, Kuo WL, Pinkel D, Albertson D, CordonCardo C, Jain AN, Waldman FM: Array-based comparative genomic hybridization for genome-wide screening of DNA copy number in bladder tumors. Cancer Res 2003, 63:2872-2880

26. Angrand PO, Apiou F, Stewart AF, Dutrillaux B, Losson R, Chambon P: NSD3, a new SET domain-containing gene, maps to 8p12 and is amplified in human breast cancer cell lines. Genomics 2001, 74 : 79-88

27. Bergemann AD, Cole F, Hirschhorn K: The etiology of WolfHirschhorn syndrome. Trends Genet 2005, 21:188-195

28. Morishita M, di Luccio E: Cancers and the NSD family of histone lysine methyltransferases. Biochim Biophys Acta 2011, 1816: $158-163$

29. Takeuchi M, Harigai M, Momohara S, Ball E, Abe J, Furuichi K, Kamatani N: Cloning and characterization of DPPL1 and DPPL2, representatives of a novel type of mammalian phosphatidate phosphatase. Gene 2007, 399:174-180

30. Saha S, Bardelli A, Buckhaults P, Velculescu VE, Rago C, St Croix B, Romans KE, Choti MA, Lengauer C, Kinzler KW, Vogelstein B: A phosphatase associated with metastasis of colorectal cancer. Science 2001, 294:1343-1346

31. Tonks NK, Muthuswamy SK: A brake becomes an accelerator: pTP1B-a new therapeutic target for breast cancer. Cancer Cell 2007, 11:214-216

32. Zhang ZY: Protein tyrosine phosphatases: prospects for therapeutics. Curr Opin Chem Biol 2001, 5:416-423

33. Barretina J, Caponigro G, Stransky N, Venkatesan K, Margolin AA, Kim S, et al: The Cancer Cell Line Encyclopedia enables predictive modelling of anticancer drug sensitivity. Nature 2012, 483:603-607

34. Cancer Genome Atlas Research Network: Comprehensive genomic characterization of squamous cell lung cancers. Nature 2012, 489: $519-525$

35. Bashyam MD, Bair R, Kim YH, Wang P, Hernandez-Boussard T, Karikari CA, Tibshirani R, Maitra A, Pollack JR: Array-based comparative genomic hybridization identifies localized DNA amplifications and homozygous deletions in pancreatic cancer. Neoplasia 2005, 7:556-562

36. Aguirre AJ, Brennan C, Bailey G, Sinha R, Feng B, Leo C, Zhang Y, Zhang J, Gans JD, Bardeesy N, Cauwels C, Cordon-Cardo C, 
Redston MS, DePinho RA, Chin L: High-resolution characterization of the pancreatic adenocarcinoma genome. Proc Natl Acad Sci U S A 2004, 101:9067-9072

37. Pole JC, Courtay-Cahen C, Garcia MJ, Blood KA, Cooke SL, Alsop AE, Tse DM, Caldas C, Edwards PA: High-resolution analysis of chromosome rearrangements on $8 \mathrm{p}$ in breast, colon and pancreatic cancer reveals a complex pattern of loss, gain and translocation. Oncogene 2006, 25:5693-5706

38. Peifer M, Fernández-Cuesta L, Sos ML, George J, Seidel D, Kasper LH, et al: Integrative genome analyses identify key somatic driver mutations of small-cell lung cancer. Nat Genet 2012, 44: $1104-1110$

39. Yang ZQ, Liu G, Bollig-Fischer A, Giroux CN, Ethier SP: Transforming properties of 8p11-12 amplified genes in human breast cancer. Cancer Res 2010, 70:8487-8497

40. Sawey ET, Chanrion M, Cai C, Wu G, Zhang J, Zender L, Zhao A, Busuttil RW, Yee H, Stein L, French DM, Finn RS, Lowe SW, Powers S: Identification of a therapeutic strategy targeting amplified FGF19 in liver cancer by Oncogenomic screening. Cancer Cell 2011, 19:347-358

41. Kao J, Pollack JR: RNA interference-based functional dissection of the $17 q 12$ amplicon in breast cancer reveals contribution of coamplified genes. Genes Chromosomes Cancer 2006, 45:761-769

42. Ray ME, Yang ZQ, Albertson D, Kleer CG, Washburn JG, Macoska JA, Ethier SP: Genomic and expression analysis of the 8p11-
12 amplicon in human breast cancer cell lines. Cancer Res 2004, 64: 40-47

43. Reis-Filho JS, Simpson PT, Turner NC, Lambros MB, Jones C, Mackay A, Grigoriadis A, Sarrio D, Savage K, Dexter T, Iravani M, Fenwick K, Weber B, Hardisson D, Schmitt FC, Palacios J, Lakhani SR, Ashworth A: FGFR1 emerges as a potential therapeutic target for lobular breast carcinomas. Clin Cancer Res 2006, 12: 6652-6662

44. Bentires-Alj M, Neel BG: Protein-tyrosine phosphatase 1B is required for HER2/Neu-induced breast cancer. Cancer Res 2007, 67: 2420-2424

45. Johnson KJ, Peck AR, Liu C, Tran TH, Utama FE, Sjolund AB, Schaber JD, Witkiewicz AK, Rui H: PTP1B suppresses prolactin activation of Stat5 in breast cancer cells. Am J Pathol 2010, 177: 2971-2983

46. Julien SG, Dube N, Read M, Penney J, Paquet M, Han Y, Kennedy BP, Muller WJ, Tremblay ML: Protein tyrosine phosphatase 1B deficiency or inhibition delays ErbB2-induced mammary tumorigenesis and protects from lung metastasis. Nat Genet 2007, 39: $338-346$

47. Van Cutsem E, Kohne CH, Hitre E, Zaluski J, Chang Chien CR, Makhson A, D'Haens G, Pinter T, Lim R, Bodoky G, Roh JK, Folprecht G, Ruff P, Stroh C, Tejpar S, Schlichting M, Nippgen J, Rougier P: Cetuximab and chemotherapy as initial treatment for metastatic colorectal cancer. N Engl J Med 2009, 360:1408-1417 